\title{
Die Abklärung der Lübecker Säuglingstuberkulose
}

\section{H. L. Rieder}

\section{Clarification of the Luebeck Infant Tuberculosis}

Im Jahre 1930 erschütterte eine tödliche Tuberkuloseepidemie bei Säuglingen in Lübeck die Weltöffentlichkeit und stellte die Sicherheit der BCG-Schutzimpfung, das erste entdeckte Mittel zur Bekämpfung der Tuberkulose, scheinbar infrage. Dieser Artikel hat zum Ziel, die Essenz dieser Epidemie zusammenzufassen und den involvierten Experten Tribut zu zahlen für ein Meisterstück rigoroser wissenschaftlicher Abklärung und exemplarisch fachübergreifender Zusammenarbeit.

\section{Herstellung und Verteilung von BCG}

Calmette und Guérin entwickelten den Impfstoff BCG (Bacille Calmette-Guérin) zwischen 1908 und 1921 in 230 Subkulturen auf einem Kartoffel-Rindergalle-Medium eines Stammes von Mycobacterium bovis, der 1921 ohne jegliche Tierpathogenität und als vollständig attenuiert befunden wurde [1].

Der Impfstoff wurde 1921 erstmals durch den Pädiater WeillHallé in Paris bei einem Säugling verwendet und anschließend zuerst in Frankreich und dann in einer steigenden Anzahl europäischer Länder verwendet [2]. Auf schriftliches Verlangen wurden dem entsprechenden Arzt drei versiegelte Ampullen mit dem Impfstoff gratis zur Verfügung gestellt. Die Instruktionen zur Aufbewahrung der Impfkulturen waren klar: ,... L'étuve qui les contient ne recevera aucun autre microbe, surtout aucun autre bacille tuberculeux ..." (... Der die Kulturen enthaltende Brutschrank darf keine anderen Mikroorganismen enthalten, insbesondere aber keine anderen Tuberkulosebakterien ...) [3].
Für die Impfung wurde die orale Verabreichung von drei Dosen im Abstand von zwei Tagen innerhalb der ersten 10 Lebenstage anempfohlen, aufgenommen in einem Esslöffel körperwarmer Milch [3].

Die ersten Berichte über die Einführung von BCG in Deutschland gehen auf das Jahr 1925 in Eifel durch Buschmann, 1927 in Neukölln-Berlin durch Zadek, und 1928 in Breslau durch Prausnitz zurück [2]. Im Allgemeinen Krankenhaus Lübeck wurde das erste Kind am 10. Dezember 1929 mit dem im zugehörigen Laboratorium sub-kultivierten Impfstoff geimpft.

\section{Die Epidemie}

Im Zeitraum vom 10. Dezember 1929 bis zum 30 . April 1930 wurden in Lübeck 412 Kinder geboren, von denen 251 (inklusive drei auswärts geborene) geimpft wurden (Tab.1) [4]. Von den 251 Geimpften verstarben 77, davon 72 (28,7\% der Geimpften) mit oder an ausgedehnter autoptisch nachgewiesener Tuberkulose $[4,5]$. Von den drei bis zum 10. Februar 1930 geimpften Kindern starb keines [6]. Die Epidemie der Todesfälle erreichte ihren Höhepunkt im Juni 1930 (Abb.1) [4]. Die mediane Überlebenszeit der 72 mit oder an Tuberkulose verstorbenen Säuglinge betrug 90 Tage (Abb. 2) [4]. Alle verstarben innerhalb des ersten Lebensjahres.

Bei 126 der 174 überlebenden Kinder wurden in den radiologischen Verlaufskontrollen in den Jahren 1932 und 1933 einwandfrei Verkalkungen der Mesenteriallymphknoten nachgewiesen.

Rubrikherausgeber: R. Kropp (federführend), U. Costabel, H. S. Fuchs, H. Jungbluth, H. J. Klippe, N. Konietzko, R. Loddenkemper, G. Neumann, M. Teschner

Institutsangaben

International Union Against Tuberculosis and Lung Disease

Danksagung

Herr Uwe Molkentin stellte Arbeiten aus dem Reichsgesundheitsamt zur Verfügung und Herr Dr. Robert Kropp verschaffte zusätzliche historische Unterlagen aus den Archiven in Lübeck.

Korrespondenzadresse

PD Dr. med. Hans L. Rieder · Jetzikofenstr. 12 · 3038 Kirchlindach · Schweiz · E-mail: TBRieder@tbrieder.org

Bibliografie

Pneumologie 2003; 57: 402-405 @ Georg Thieme Verlag Stuttgart • New York • ISSN 0934-8387 
Tab. 1 Sterblichkeit von 415 Säuglingen entsprechend Impfstatus, Lübeck, 10. Dezember 1929 bis 30. April 1930 [4]

\begin{tabular}{|llll|}
\hline Impfung & $\begin{array}{l}\text { verstorben } \\
\text { ja }\end{array}$ & nein & total \\
\hline ja & 77 & 174 & 251 \\
\hline nein & 19 & 145 & 164 \\
\hline total & 96 & 319 & 415 \\
\hline
\end{tabular}

* RR (95\% VB) 2,6 (1,7-4,2)

* RR: Relatives Risiko; 95\% VB: 95\% Vertrauensbereich

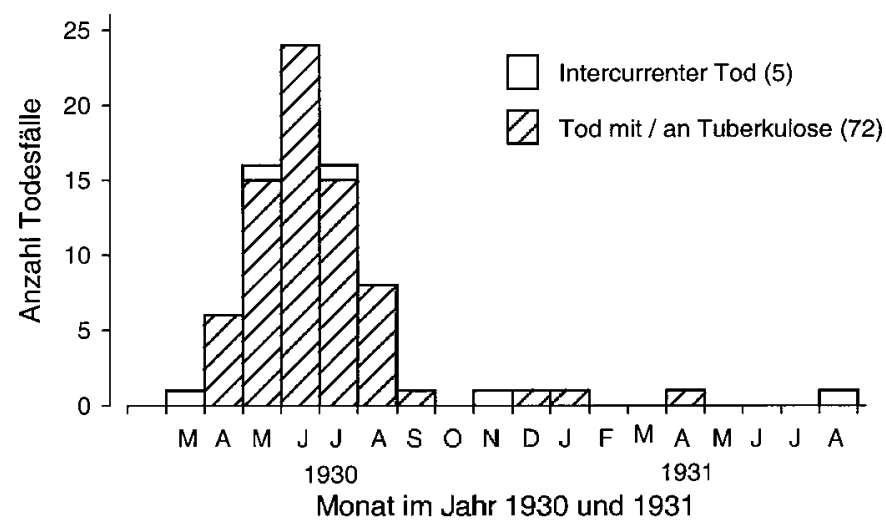

Abb. 1 Epidemiekurve der 77 Todesfälle bei Lübecker Säuglingen nach BCG-Impfung, 1930 - 1931 [4].

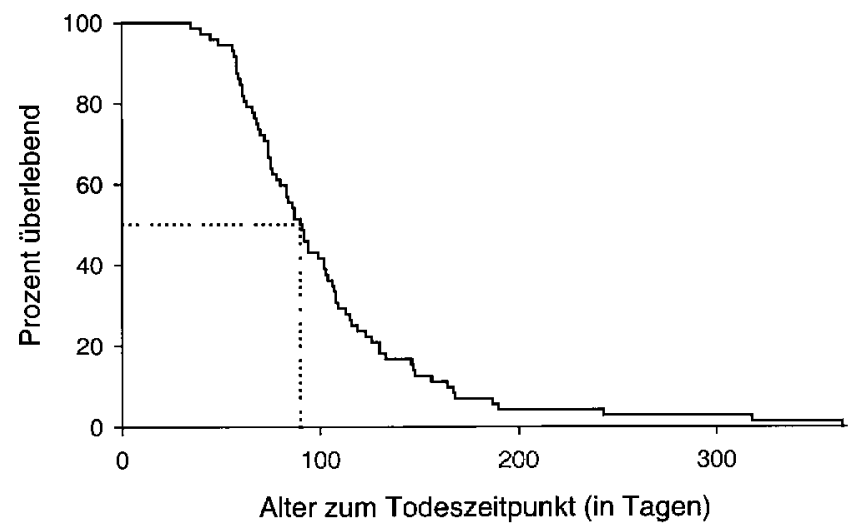

Abb. 2 Überlebenskurve der an oder mit Tuberkulose gestorbenen 72 Säuglinge, Lübeck, $1930-1931$ [4].

\section{Die Abklärung der Epidemie}

Die Gebrüder Lange (Ludwig Lange von der Bakteriologischen Abteilung des Reichsgesundheitsamtes und Bruno Lange von der Seuchenabteilung des Instituts „Robert Koch“) wurden mit der Abklärung der Epidemie beauftragt und kamen erstmals am 14. März 1930 zur Probenentnahme [7] und am 15. Mai 1930 zur weiteren Abklärung nach Lübeck [6-8]. Für die Erstellung des Datensatzes und dessen abschließender Analyse konnte Albert Moegling von der Deutschen Forschungsanstalt für Tuberkulose und der Bakteriologischen Abteilung des Reichsgesundheitsam- tes von anfangs Juli bis Ende September 1930 gewonnen werden, dessen Arbeit von Kurt Jannasch, dem Leiter der lokalen Tuberkulosefürsorge, akribisch weitergeführt wurde [4]. Die pathologische Aufarbeitung wurde durch Hans Kleinschmidt koordiniert [5].

Bereits am 17.Juli 1930 lag ein vorläufiger Bericht von Ludwig Lange vor, der die entscheidende Frage beantwortete [6]. Im Vordergrund der Abklärung stand natürlich die Frage, ob der von Petroff früher erhobene Verdacht, dass BCG seine Virulenz zurückgewinnen könnte [9], sich bewahrheitet hatte. Petroff hatte anscheinend eine morphologisch nachweisbare Dissoziation von BCG in einen avirulenten und einen virulenten Stamm aufgezeigt. Bruno Lange erhielt den implizierten BCG-Stamm von Petroff persönlich und konnte zweifelsfrei nachweisen, dass diesem virulentes $M$. tuberculosis beigemischt war [8]. Trotzdem war die Rückbildung von BCG zur Virulenz in Lübeck die wichtigste zu beantwortende Fragestellung.

Dass BCG allein als unwahrscheinlich für die Epidemie verantwortlich sein konnte, ergab sich aus der stark variierenden Letalität je nach Datum der Verabreichung des Impfstoffes: beispielsweise verstarben 17 und erkrankten 5 erheblich von den 24 Säuglingen, die vom 25. bis 27. März 1930 geimpft wurden, jedoch verstarb keiner von den 20 Säuglingen, die vom 1. bis 5. April geimpft wurden. Aus diesen epidemiologischen Hinweisen ergab sich die Hypothese, dass der täglich verwendete Impfstoff aus einer wechselnden Mischung von virulentem und avirulentem Material bestand [4]. Da nach der Realisierung der Epidemie die vorhandenen Impfstoffröhrchen bis auf wenige Impfstoffreste [7] vernichtet worden waren, konnte diese Hypothese nicht systematisch geprüft werden.

Unter den Impfstoffresten konnten bei acht keine lebenden Mykobakterien gezüchtet werden, bei einem wurde nur BCG nachgewiesen, bei einem weiteren nur virulentes $M$. tuberculosis der „Kiel“-Gruppe [7]. Bei Organkulturen von 30 gestorbenen und einer Magensaftkultur eines lebenden Kindes wurden in 28 Fällen für Meerschweinchen pathogenes, für Kaninchen jedoch apathogenes M. tuberculosis isoliert, alle Stämme vom gleichen Typus.

\section{Der Stamm „Werner“ aus Kiel}

Den M. tuberculosis-Stamm „Werner“ hatte das Kieler Hygienische Institut vom Institut Robert Koch, Berlin, bezogen; er wurde am 9. September 1929 an das Laboratorium in Lübeck auf dessen Anfrage gesandt [7], nachdem infolge eines Thermostatenfehlers im Sommer sämtliche Kulturen vernichtet worden waren [10]. Dieser Stamm zeigte glücklicherweise ein seltenes Charakteristikum, das seine relativ leichte Identifizierung erlaubte, nämlich die Tendenz dieses Stammes zur „Vergrünung“. Nach wenigen Tagen Inkubation trat ein leichter Grünstich auf, der seine stärkste Ausprägung nach zwei bis vier Wochen entwickelte. Dieser „Blaugrünstich“ war ein relativ seltenes Merkmal und ließ daher eine eindeutige Zuordnung der in Lübeck isolierten Stämme zu. Von 200 verschiedenen im Laboratorium für Tuberkuloseforschung des Reichsgesundheitsamts untersuchten humanen und bovinen Stämmen zeigten außer den Kielstämmen nur zwei eine solche Vergrünung und BCG-Stämme nie. 
einheit mit fahrlässiger Körperverletzung zu zwei Jahren Ge-

Im Laboratorium des Lübecker Krankenhauses wurden sowohl BCG als auch der Stamm „Werner“ im gleichen Brutschrank propagiert [10], ein deutlicher Verstoß gegen die von Calmette geforderte Sorgsamspflicht.

Nach Angabe der Laboratoriumsschwester wurde für die Herstellung des Impfstoffes von Kulturmaterial etwa 14 Tage alter Eiernährböden ausgegangen. Es wurde im Mörser fein zerrieben, durch Zusetzen von Traubenzucker-Glyzerinlösung emulgiert und die aufbereitete Emulsion in einer Packung von je drei $2 \mathrm{ml}$ enthaltenden Fläschchen für jedes Kind an die Hebammen abgegeben [5]. Wie die eigentliche Kontamination von BCG mit M. tuberculosis zustande kam, konnte nicht einwandfrei geklärt werden.

Um das Schlucken der Impflösung zu erleichtern, wurde den Kindern von einzelnen Hebammen die Nase zugehalten. Dies erklärt das Entstehen von Aspirations-Lungentuberkulosen und tuberkulösen Mittelohrentzündungen, die bei mehreren Kindern festgestellt wurden. Des Weiteren wurde der Impfstoff entgegen den Vorschriften nicht immer in einem Esslöffel mit körperwarmer Milch abgegeben, sondern gelegentlich auch mit anderen Flüssigkeiten, deren Quantität zudem in hohem Maße variierte (vom Esslöffel bis zum Beifügen des Impfstoffs zum Gesamtinhalt der Flaschenmahlzeit), vermischt und gelegentlich aufgehitzt.

\section{Zusammenfassende Beurteilung der Experten}

Epidemiologisch sprach die Abklärung für eine Beimischung von virulentem Material zum BCG-Impfstoff in variablen Mischverhältnissen [4].

Die bakteriologische Abklärung identifizierte den Stamm „Werner" aus Kiel als den virulenten Anteil [7].

Die pathologisch-klinische Abklärung zeigte massiven Mesenterialbefall und Disseminierung [5]. Otitiden und Aspirationstuberkulosen [8] wiesen auf das Problem unsachgemäßer Fütterung (unter Zuhalten der Nase) hin. Die relativ niedrige Letalität wurde auf die variable Virulenz des Kieler Stammes, die unterschiedlichen Verdünnungsmaßnahmen, Erhitzungen des den Impfstoff erhaltenden Einnahmemittels, sowie postprandiales Erbrechen und nicht aufgenommene Bodensätze aus Flaschennahrung zurückgeführt.

\section{Gerichtliches Nachspiel}

Vom 12. Oktober 1931 bis zum 6. Februar 1932 fand die gerichtliche Untersuchung vor der II. Großen Strafkammer des Landgerichts der Freien und Hansestadt Lübeck und des oldenburgischen Landesteils Lübeck statt [10]. Zu verantworten hatten sich der Chefarzt der Inneren Medizin (gleichzeitig Verantwortlicher des Laboratoriums), der ärztliche Leiter des Gesundheitsamtes, der Chefarzt der Kinderklinik und die Laborschwester. Der erstere wurde wegen eines Vergehens der fahrlässigen Tötung in Tat-

fängnis verurteilt. Der ärztliche Leiter des Gesundheitswesens wurde wegen desselben Vergehens zu einer Gefängnisstrafe von einem Jahr und drei Monaten verurteilt. Der Chefarzt der Kinderklinik wurde freigesprochen und die Laborschwester aus Mangel an Beweisen freigesprochen.

\section{Epilog}

In einer bemerkenswerten Qualität und Zusammenarbeit von Epidemiologie, Bakteriologie und Pathologie wurde die Lübecker Epidemie in kurzer Zeit abgeklärt und BCG vollständig rehabilitiert. Trotzdem wurde die generelle Einführung der BCG-Impfung in Deutschland dadurch verzögert.

Die BCG-Impfung wurde weltweit erst in den 50er-Jahren als allgemeine Schutzimpfung eingeführt [11]. Sie verleiht einen relativ guten Schutz gegen disseminierte Formen der Tuberkulose (vor allem die tuberkulöse Meningitis) im Kleinkindesalter [12], trägt aber wenig zur Verbesserung der epidemiologischen Entwicklung der Tuberkulose bei [13]. Eine zunehmende Anzahl industrialisierter Länder hat die allgemeine BCG-Impfung inzwischen sistiert [14], da die Indikation zum Schutz gegen die tuberkulöse Meningitis mit der Verbesserung der epidemiologischen Lage nicht mehr gegeben ist und die seltenen wichtigen Impfkomplikationen (Osteomyelitis und generalisierte Erkrankung) in einigen Ländern häufiger wurden als die Krankheit, die die Impfung verhüten sollte [12].

Dass Kreuzkontaminationen ein leider nicht seltenes Geschehen in mykobakteriologischen Laboratorien sind, ist inzwischen hinreichend etabliert [15]. Calmette hatte dies befürchtet und entsprechende Anweisungen in aller Deutlichkeit gegeben, Empfehlungen, die sowohl vom internistischen Chefarzt des Lübecker Krankenhauses als auch der zuständigen Gesundheitsbehörde fahrlässig und mit katastrophalen Folgen missachtet wurden.

\section{Literatur}

${ }^{1}$ Calmette A. Preventive vaccination against tuberculosis with BCG. Proc Roy Soc Med 1931; 24: 85-94

${ }^{2}$ Institut Pasteur. Vaccination préventive de la tuberculose de l'homme et des animaux par le BCG. Rapports et documents provenant des divers pays (France exceptée) transmis à l'Institut Pateur en 1932.1 ed. Paris: Masson et Cie, 1932: 1-366

${ }^{3}$ Calmette A, Guérin C, Nègre L et al. Prémunition des nouveau-nés contre la tuberculose par le vaccin BCG (1921 à 1926). Ann Inst Pasteur 1926; 40: 89-134

${ }^{4}$ Moegling A. Die „Epidemiologie“ der Lübecker Säuglingstuberkulose. Arbeiten a d Reichsges-Amt 1935; 69: 1-24

${ }^{5}$ Schürmann P, Kleinschmidt H. Pathologie und Klinik der Lübecker Säuglingstuberkuloseerkrankungen. Arbeiten a d Reichsges-Amt 1935; 69: $25-204$

${ }^{6}$ Lange L. Zu den Tuberkuloseschutzimpfungen in Lübeck. Zeitschr Tuberkulose 1930; 57: $305-310$

${ }^{7}$ Lange L, Pescatore H. Bakteriologische Untersuchungen zur Lübecker Säuglingstuberkulose. Arbeiten a d Reichsges-Amt 1935; 69: 205-305

${ }^{8}$ Lange B. Untersuchungen zur Klärung der Ursachen der im Anschluss an die Calmette-Impfung aufgetretenen Säuglingserkrankungen in Lübeck. Zeitschr Tuberkulose 1930; 59: 1-18 
${ }^{9}$ Petroff SA, Branch A, Steenken Jr W. A study of Bacillus Calmette-Guérin (BCG). I. Biological characteristics, cultural „dissociation“ and animal experimentation. Am Rev Tuberc 1929; 19: 9-46

${ }^{10}$ Edelhoff J. Der Calmette-Prozess. Der Wagen. Ein Lübeckisches Jahrbuch. Lübeck: Hansisches Verlagskontor, 1984: 62-68

${ }^{11}$ Comstock GW. The international tuberculosis campaign: a pioneering venture in mass vaccination and research. Clin Infect Dis 1994; 19: $528-540$
${ }^{12}$ Rieder HL. Interventions for tuberculosis control and elimination. Paris: International Union Against Tuberculosis and Lung Disease, 2002: $1-251$

${ }^{13}$ Styblo K, Meijer J. Impact of BCG vaccination programmes in children and young adults on the tuberculosis problem. Tubercle 1976; 57 : $17-43$

14 Trnka L, Dankova D, Zitova J et al. Survey of BCG vaccination policy in Europe: 1994-96. Bull World Health Organ 1998; 76: 85-91

${ }^{15}$ Heifets L. False diagnosis of tuberculosis. (Editorial). Int J Tuberc Lung Dis 2001; 5: 789-790

\section{Buchbesprechung}

\section{Die Hosen des Pythagoras - Physik, Gott und die Frauen Margaret Wertheim}

Aus dem Englischen von Karin Schuler, Karin Miedler, Silke Egelhof Piper Verlag GmbH, München, Originalausgabe 1994; ungekürzte Taschenbuchausgabe 2000, $386 \mathrm{~S}$. , $17 \mathrm{Abb}$. 10,90. ISBN 3-492-22923-9

Pythagoras von Samos (582-507 v.Chr.) - uns allen vom Lehrsatz des rechteckigen Dreiecks ${ } \mathrm{c}^{2}=\mathrm{a}^{2}+\mathrm{b}^{2 \text { “ }}$ bekannt - begründete eine sozialreformerische Philosophenschule in Kroton/Unteritalien. Er verstand die Grundbegriffe des „Seins“ nicht in stofflichen „Elementen (Erde, Feuer, Luft, Wasser)“ sondern in der immateriellen Magie der Zahlen. Als Monotheist sah er in Gott den Mathematiker, der die Sphärenharmonie bestimmt und den Mechanismus im Denken erschaffen hatte.

Nach 15 Jahren Studium in Babylon und fünf Jahren in Ägypten lehnte Pythagoras die konventionelle griechische Bart- und Haartracht ab und trug statt wallender Gewänder die „persischen Hosen“ - die nun als „Aufreißer“ für den Buchtitel dienen. Die Pythagoräer („mathematikoi“) verstanden sich als Priesterschaft, dienten einer Philosophie von Rationalität und Mystik und schufen das Prinzip des „mathematischen Mannes“.

Als Nonkonformist gab Pythagoras auch Frauen Zugang zu seiner Gemeinschaft: seine Ehefrau Theano unterrichtete Mathematik und Kosmologie; als frühe Schülerinnen gelten Phintys, Melissa und Tymicha. Und auch Platon (424-347 v. Chr.) ließ Frauen an seiner Akademie zu. Als bedeutendste Interpretin der neoplatonischen Schule gilt Hypathia, Mathematikerin und Erfinderin des Astrolabiums. Sie lehnte den christlichen Glauben ab und ist deshalb in Alexandria $425 \mathrm{n}$. Chr. gelyncht worden.

Geprägt von Aristotels' (384-322 v.Chr.) Geringschätzung der Frauen, der schroffen Ablehnung der „Ecclesia“, René Descantes' (1596-1657) Postulat „Die Naturwissenschaften bleiben - wie auch die Heilige Schrift - den Männern vorbehalten“; eine Domäne allein des „Mathematischen Mannes“. Auch durch Isaac Newtons (1642 - 1727) Vergleich, dass die Männer als Planeten gelten, die Frauen nur als Monde deren Umlaufbahnen beibehalten dürfen, erwies sich der „kleine Unterschied“ als großes Hindernis für die Frauen. Mit Vorurteilen und Befremden begegnete man ihrem Ansinnen, sich mit der Physik, der „Katholischen Kir- che der Wissenschaft“, der „Domäne des Mannes“ überhaupt beschäftigen zu wollen - als „Wissenschaftsgefährtinnen“ waren sie dagegen wohl gelitten! Bis ins späte 18. Jh. sind sie weder von der Royal Society in London noch von der Académie Française in Paris akzeptiert worden.

Doch die intelligenten und klugen Frauen setzten sich mit lustgetriebener Neugier auf ihrem steinigen Wege durch! Nicht etwa in einer komödiantischen Lysistrata-Verweigerung à la Aristophanes (450-385 v.Chr.) - vom 17. bis zur Mitte des 20.Jh. stürmten sie geduldig und strebsam die „Männerbastionen“. Beispielhaft stehen u.a. die Astronomin Maria Winkelmann (1670-1720; Berlin), Voltaires Vertraute, die Mathematikerin Emilie du Châtelet (1706 - 1749; Paris), die Physikerin Mary Sommerville (1780 - 1872; Cambridge), die polnische Physikerin Marie Curie (1867-1934, Nobelpreis 1904; Paris), Otto Hahns Assistentin, die Physikerin Lise Meitner (1878-1968; Berlin), Albert Einsteins Mitarbeiterin Emmy Noether („Noether-Theorem“; 1882 - 1935; Göttingen/USA). Und viele andere, deren Namen und Leistungen uns weniger bekannt sind wie z.B. Oppenheimers Mitarbeiterin, die Teilchenphysikerin Chien-Shiung Wu (geb. 1912; USA) und die Nobelpreisträgerin 1963 für die Schalentheorie des Atomkerns, Maria Göppert-Mayer (1906-1972; Göttingen/USA) - sie alle „bürdeten sich freiwillig die Last des Mannes auf“.

Die heutigen „Feministinnen“ sind zwar immer noch nicht zufrieden, doch zeigt die Entwicklung deutliche Besserungen: z.B. in Deutschland ist der Anteil der Frauen an Hochschulen 1999 auf $45,2 \%$ gestiegen und immer mehr junge Frauen entscheiden sich für technische Fächer (in den Ingenieurwissenschaften waren es $21,8 \%$ ) und $30 \%$ der Doktoranden waren Frauen - ein „Ersatzschauplatz für die Geschlechterkontroverse"?

Die Autorin hat Physik, Mathematik und Informatik studiert und tritt in ihrem sensibel recherchierten, auf umfassendem Literaturstudium beruhendem Werke fachlich kompetent, witzig und klug für die „Frauen in der Physik“ ein: sie haben mehr erreicht als sie je erträumt hatten! In einem Anhang werden Zitate detailliert belegt; eine Auswahlbibliographie und ein Register runden eine überaus spannende Lektüre $a b$.

Heinz S. Fuchs, Bad Godesberg 